\title{
Some characterizations for SOC-monotone and SOC-convex functions
}

\author{
Jein-Shan Chen • Xin Chen • Shaohua Pan • \\ Jiawei Zhang
}

Received: 29 June 2007 / Accepted: 21 October 2008 / Published online: 7 November 2008

C) Springer Science+Business Media, LLC. 2008

\begin{abstract}
We provide some characterizations for SOC-monotone and SOC-convex functions by using differential analysis. From these characterizations, we particularly obtain that a continuously differentiable function defined in an open interval is SOC-monotone (SOC-convex) of order $n \geq 3$ if and only if it is 2-matrix monotone (matrix convex), and furthermore, such a function is also SOC-monotone (SOC-convex) of order $n \leq 2$ if it is 2-matrix monotone (matrix convex). In addition, we also prove that Conjecture 4.2 proposed in Chen (Optimization 55:363-385, 2006) does not hold in general. Some examples are included to illustrate that these characterizations open convenient ways to verify the SOCmonotonicity and the SOC-convexity of a continuously differentiable function defined on an open interval, which are often involved in the solution methods of the convex second-order cone optimization.
\end{abstract}

Keywords Second-order cone $\cdot$ SOC-monotone function $\cdot$ SOC-convex function

Mathematics Subject Classification (2000) $\quad 26 \mathrm{~A} 48 \cdot 26 \mathrm{~A} 51 \cdot 26 \mathrm{~B} 05 \cdot 90 \mathrm{C} 25$

\footnotetext{
J.-S. Chen $(\varangle)$

Department of Mathematics, National Taiwan Normal University, Taipei 11677, Taiwan e-mail: jschen@math.ntnu.edu.tw
}

\section{Chen}

Department of Industrial and Enterprise System Engineering, University of Illinois at Urbana-Champaign, Urbana 61801, IL, USA

e-mail: xinchen@uiuc.edu

S. Pan

School of Mathematical Sciences, South China University of Technology, Guangzhou 510640, China e-mail: shhpan@scut.edu.cn

\section{J. Zhang}

Department of Information, Operations and Management Sciences, New York University, New York 10012-1126, NY, USA

e-mail: jzhang@stern.nyu.edu 


\section{Introduction}

The second-order cone (SOC) in $\mathbb{R}^{n}$, also called the Lorentz cone, is a set defined by

$$
\mathcal{K}^{n}:=\left\{\left(x_{1}, x_{2}\right) \in \mathbb{R} \times \mathbb{R}^{n-1} \mid\left\|x_{2}\right\| \leq x_{1}\right\},
$$

where $\|\cdot\|$ denotes the Euclidean norm, and $\mathcal{K}^{1}$ denotes the set of nonnegative reals $\mathbb{R}_{+}$. It is known that $\mathcal{K}^{n}$ is a closed convex self-dual cone with nonempty interior $\operatorname{int}\left(\mathcal{K}^{n}\right)$. For any $x, y \in \mathbb{R}^{n}$, we write $x \succeq_{\mathcal{K}^{n}} y$ if $x-y \in \mathcal{K}^{n}$; and write $x \succ_{\mathcal{K}^{n}} y$ if $x-y \in \operatorname{int}\left(\mathcal{K}^{n}\right)$. In other words, we have $x \succeq_{\mathcal{K}^{n}} 0$ if and only if $x \in \mathcal{K}^{n}$ and $x \succ_{\mathcal{K}^{n}} 0$ if and only if $x \in \operatorname{int}\left(\mathcal{K}^{n}\right)$. The relation $\succeq_{\mathcal{K}^{n}}$ is a partial ordering, but not a linear ordering in $\mathcal{K}^{n}$, i.e., there exist $x, y \in \mathcal{K}^{n}$ such that neither $x \succeq_{\mathcal{K}^{n}} y$ nor $y \succeq_{\mathcal{K}^{n}} x$. To see this, let $x=(1,1), y=(1,0)$, and then we have $x-y=(0,1) \notin \mathcal{K}^{2}, y-x=(0,-1) \notin \mathcal{K}^{2}$.

For any $x=\left(x_{1}, x_{2}\right), y=\left(y_{1}, y_{2}\right) \in \mathbb{R} \times \mathbb{R}^{n-1}$, we define their Jordan product as

$$
x \circ y=\left(\langle x, y\rangle, y_{1} x_{2}+x_{1} y_{2}\right) .
$$

we write $x^{2}$ to mean $x \circ x$ and write $x+y$ to mean the usual componentwise addition of vectors. Then $\circ,+$, and $e=(1,0, \ldots, 0)^{T} \in \mathbb{R}^{n}$ have the following basic properties (see [7,8]): (1) $e \circ x=x$ for all $x \in \mathbb{R}^{n}$. (2) $x \circ y=y \circ x$ for all $x, y \in \mathbb{R}^{n}$. (3) $x \circ\left(x^{2} \circ y\right)=x^{2} \circ(x \circ y)$ for all $x, y \in \mathbb{R}^{n}$. (4) $(x+y) \circ z=x \circ z+y \circ z$ for all $x, y, z \in \mathbb{R}^{n}$. Note that the Jordan product is not associative. Besides, $\mathcal{K}^{n}$ is not closed under Jordan product.

We recall from $[7,8]$ that each $x=\left(x_{1}, x_{2}\right) \in \mathbb{R} \times \mathbb{R}^{n-1}$ admits a spectral factorization, associated with $\mathcal{K}^{n}$, of the form

$$
x=\lambda_{1}(x) \cdot u_{x}^{(1)}+\lambda_{2}(x) \cdot u_{x}^{(2)},
$$

where $\lambda_{1}(x), \lambda_{2}(x)$ and $u_{x}^{(1)}, u_{x}^{(2)}$ are the spectral values and the associated spectral vectors of $x$ given by

$$
\lambda_{i}(x)=x_{1}+(-1)^{i}\left\|x_{2}\right\|, \quad u_{x}^{(i)}=\frac{1}{2}\left(1,(-1)^{i} \bar{x}_{2}\right) \text { for } i=1,2,
$$

with $\bar{x}_{2}=\frac{x_{2}}{\left\|x_{2}\right\|}$ if $x_{2} \neq 0$ and otherwise $\bar{x}_{2}$ being any vector in $\mathbb{R}^{n-1}$ such that $\left\|\bar{x}_{2}\right\|=1$. If $x_{2} \neq 0$, the factorization is unique. By the spectral factorization, for any $f: \mathbb{R} \rightarrow \mathbb{R}$, we can define a vector-valued function associated with $\mathcal{K}^{n}(n \geq 1)$ by

$$
f^{\mathrm{soc}}(x)=f\left(\lambda_{1}(x)\right) u_{x}^{(1)}+f\left(\lambda_{2}(x)\right) u_{x}^{(2)}, \quad \forall x=\left(x_{1}, x_{2}\right) \in \mathbb{R} \times \mathbb{R}^{n-1},
$$

and call it the SOC-function induced by $f$. If $f$ is defined only on a subset of $\mathbb{R}$, then $f^{\mathrm{soc}}$ is defined on the corresponding subset of $\mathbb{R}^{n}$. The definition is unambiguous whether $x_{2} \neq 0$ or $x_{2}=0$. The cases of $f^{\mathrm{soc}}(x)=x^{1 / 2}, x^{2}, \exp (x)$ were discussed in [7]. In fact, the above definition (5) is analogous to one associated with the semidefinite cone; see $[19,20]$.

Recently, the concepts of SOC-monotone and SOC-convex functions are introduced in [5]. Especially, a function $f: J \rightarrow \mathbb{R}$ with $J \subseteq \mathbb{R}$ is said to be $S O C$-monotone of order $n$ if

$$
x \succeq_{\mathcal{K}^{n}} y \Longrightarrow f^{\mathrm{soc}}(x) \succeq_{\mathcal{K}^{n}} f^{\mathrm{soc}}(y)
$$

for any $x, y \in \operatorname{dom} f^{\mathrm{soc}} \subseteq \mathbb{R}^{n}$, where $\operatorname{dom} f^{\mathrm{soc}}$ denotes the domain of the function $f^{\mathrm{soc}}$; and $f$ is said to be SOC-convex of order $n$ if, for any $x, y \in \operatorname{dom} f^{\text {soc }}$,

$$
f^{\mathrm{soc}}(\lambda x+(1-\lambda) y) \preceq_{\mathcal{K}^{n}} \lambda f^{\mathrm{soc}}(x)+(1-\lambda) f^{\mathrm{soc}}(y) \quad \lambda \in[0,1] .
$$


The function $f$ is said to be SOC-monotone (respectively, SOC-convex) if it is SOCmonotone of all order $n$ (respectively, SOC-convex of all order $n$ ), and $f$ is SOC-convex on $J$ if and only if $-f$ is SOC-concave on $J$. The concepts of SOC-monotone and SOC-convex functions are analogous to matrix monotone and matrix convex functions $[2,10,11,14]$, and are special cases of operator monotone and operator convex functions $[1,3,12]$. For example, the function $f$ is said to be $n$-matrix convex on $J$ if

$$
f(\lambda A+(1-\lambda) B) \preceq \lambda f(A)+(1-\lambda) f(B) \quad \lambda \in[0,1]
$$

for arbitrary Hermitian $n \times n$ matrices $A$ and $B$ with spectra in $J$. It is clear that the set of SOC-monotone functions and the set of SOC-convex functions are closed under positive linear combinations and pointwise limits.

There has been systematic study on matrix monotone and matrix convex functions, and moreover, characterizations for such functions have been explored; see $[4,10,11,13,14]$ and the references therein. To the contrast, the study on SOC-monotone and SOC-convex functions just starts its first step. One reason is that they were viewed as special cases of operator monotone and operator convex functions. However, we recently observed that SOC-monotone and SOC-convex functions play an important role in the design of solutions methods for convex second-order cone programs (SOCPs); for example, the proximal-like methods in [15] and the augmented Lagrangian method introduced in Sect. 5. On the other hand, we all know that the developments of matrix-valued functions have major contributions in the solution of optimization problems. Thus, we hope similar systematic study on SOC-functions can be exploited so that it can be readily adopted to optimization field. This is the main motivation of the paper.

Although some work was done in [5] for SOC-monotone and SOC-convex functions, the focus there is to provide some specific examples by the definition and it seems difficult to exploit the characterizations there to verify whether a given function is SOC-convex or not. In this paper, we employ differential analysis to establish some useful characterizations which will open convenient ways to verify the SOC-monotonicity and the SOC-convexity of a function defined on an open interval. Particularly, from these characterizations, we obtain that a continuously differentiable function defined on an open interval is SOC-monotone (SOCconvex) of order $n \geq 3$ if and only if it is 2-matrix monotone (matrix convex), and such a function is also SOC-monotone (SOC-convex) of order $n \leq 2$ if it is 2-matrix monotone (matrix convex). Thus, if such functions are 2-matrix monotone (matrix convex), then it must be SOC-monotone (SOC-convex). It should be pointed out that the analysis of this paper can not be obtained from those for matrix-valued functions. One of the reasons is that the matrix multiplication is associative whereas the Jordan product is not.

Throughout the paper, $\langle\cdot, \cdot\rangle$ denotes the Euclidean inner product, $\mathbb{R}^{n}$ denotes the space of $n$-dimensional real column vectors, and $\mathbb{R}^{n_{1}} \times \cdots \times \mathbb{R}^{n_{m}}$ is identified with $\mathbb{R}^{n_{1}+\cdots+n_{m}}$. Thus, $\left(x_{1}, \ldots, x_{m}\right) \in \mathbb{R}^{n_{1}} \times \cdots \times \mathbb{R}^{n_{m}}$ is viewed as a column vector in $\mathbb{R}^{n_{1}+\cdots+n_{m}}$. Also, $I$ represents an identity matrix of suitable dimension; $J$ is a subset of $\mathbb{R}$; and $\mathbf{0}$ is a zero matrix or vector of suitable dimension. The notation ${ }^{T}$ means transpose and $C^{(i)}(J)$ denotes the family of functions which are defined on $J \subseteq \mathbb{R}$ to $\mathbb{R}$ and have the $i$-th continuous derivative. For a function $f: \mathbb{R} \rightarrow \mathbb{R}, f^{(i)}(x)$ represents the $i$-th order derivative of $f$ at $x \in \mathbb{R}$, and the first-order and the second-order derivative of $f$ are also written as $f^{\prime}$ and $f^{\prime \prime}$, respectively. For any $f: \mathbb{R}^{n} \rightarrow \mathbb{R}, \nabla f(x)$ denotes the gradient of $f$ at $x \in \mathbb{R}^{n}$ and $\operatorname{dom} f$ denotes the domain of $f$. For any differentiable mapping $F=\left(F_{1}, \ldots, F_{m}\right)^{T}: \mathbb{R}^{n} \rightarrow \mathbb{R}^{m}$, $\nabla F(x)=\left[\nabla F_{1}(x) \cdots \nabla F_{m}(x)\right]$ is an $n \times m$ matrix which denotes the transposed Jacobian of $F$ at $x$. For any symmetric matrices $A, B \in \mathbb{R}^{n \times n}$, we write $A \succeq B$ (respectively, $A \succ B$ ) to mean $A-B$ is positive semidefinite (respectively, positive definite). 


\section{Preliminaries}

In this section, we develop the second-order Taylor's expansion for the vector-valued SOCfunction $f^{\mathrm{soc}}$ defined as in (5) which is crucial in our subsequent analysis. To the end, we assume that $f \in C^{(2)}(J)$ with $J$ being an open interval in $\mathbb{R}$ and $\operatorname{dom} f^{\text {soc }} \subseteq \mathbb{R}^{n}$.

Given any $x \in \operatorname{dom} f^{\text {soc }}$ and $h=\left(h_{1}, h_{2}\right) \in \mathbb{R} \times \mathbb{R}^{n-1}$, we have $x+t h \in \operatorname{dom} f^{\text {soc }}$ for any sufficiently small $t>0$. We wish to calculate the Taylor's expansion of the function $f^{\mathrm{soc}}(x+t h)$ at $x$ for any sufficiently small $t>0$. In particular, we are interested in finding matrices $\nabla f^{\mathrm{soc}}(x)$ and $A_{i}(x)$ for $i=1,2, \ldots, n$ such that

$$
f^{\mathrm{soc}}(x+t h)=f^{\mathrm{soc}}(x)+t \nabla f^{\mathrm{soc}}(x) h+\frac{1}{2} t^{2}\left[\begin{array}{c}
h^{T} A_{1}(x) h \\
h^{T} A_{2}(x) h \\
\vdots \\
h^{T} A_{n}(x) h
\end{array}\right]+o\left(t^{2}\right) .
$$

For convenience, we omit the variable notion $x$ in $\lambda_{i}(x)$ for $i=1,2$ in the discussions below.

It is known that $f^{\mathrm{soc}}$ is differentiable (respectively, smooth) if and only if $f$ is differentiable (respectively, smooth); see [6,8]. Moreover, there holds that

$$
\nabla f^{\mathrm{soc}}(x)=\left[\begin{array}{c}
b^{(1)} \\
c^{(1)} \frac{x_{2}^{T}}{\left\|x_{2}\right\|} \\
c^{(1)} \frac{x_{2}}{\left\|x_{2}\right\|} a^{(0)} I+\left(b^{(1)}-a^{(0)}\right) \frac{x_{2} x_{2}^{T}}{\left\|x_{2}\right\|^{2}}
\end{array}\right]
$$

if $x_{2} \neq 0$, and otherwise

$$
\nabla f^{\mathrm{soc}}(x)=f^{\prime}\left(x_{1}\right) I
$$

where

$$
a^{(0)}=\frac{f\left(\lambda_{2}\right)-f\left(\lambda_{1}\right)}{\lambda_{2}-\lambda_{1}}, \quad b^{(1)}=\frac{f^{\prime}\left(\lambda_{2}\right)+f^{\prime}\left(\lambda_{1}\right)}{2}, \quad c^{(1)}=\frac{f^{\prime}\left(\lambda_{2}\right)-f^{\prime}\left(\lambda_{1}\right)}{2} .
$$

Therefore, we only need to derive the formula of $A_{i}(x)$ for $i=1,2, \ldots, n$ in (8).

We first consider the case where $x_{2} \neq 0$ and $x_{2}+t h_{2} \neq 0$. By the definition (5),

$$
\begin{aligned}
& f^{\mathrm{soc}}(x+t h)=\frac{1}{2} f\left(x_{1}+t h_{1}-\left\|x_{2}+t h_{2}\right\|\right)\left[\begin{array}{c}
1 \\
-\frac{x_{2}+t h_{2}}{\left\|x_{2}+t h_{2}\right\|}
\end{array}\right] \\
& +\frac{1}{2} f\left(x_{1}+t h_{1}+\left\|x_{2}+t h_{2}\right\|\right)\left[\begin{array}{c}
1 \\
\frac{x_{2}+t h_{2}}{\left\|x_{2}+t h_{2}\right\|}
\end{array}\right] \\
& =\left[\begin{array}{c}
\frac{f\left(x_{1}+t h_{1}-\left\|x_{2}+t h_{2}\right\|\right)+f\left(x_{1}+t h_{1}+\left\|x_{2}+t h_{2}\right\|\right)}{2} \\
\frac{f\left(x_{1}+t h_{1}+\left\|x_{2}+t h_{2}\right\|\right)-f\left(x_{1}+t h_{1}-\left\|x_{2}+t h_{2}\right\|\right)}{2} \frac{x_{2}+t h_{2}}{\left\|x_{2}+t h_{2}\right\|}
\end{array}\right] \\
& :=\left[\begin{array}{l}
\Xi_{1} \\
\Xi_{2}
\end{array}\right] \text {. }
\end{aligned}
$$


To derive the Taylor's expansion of $f^{\mathrm{soc}}(x+t h)$ at $x$ with $x_{2} \neq 0$, we first write out and expand $\left\|x_{2}+t h_{2}\right\|$. Notice that

$$
\left\|x_{2}+t h_{2}\right\|=\sqrt{\left\|x_{2}\right\|^{2}+2 t x_{2}^{T} h_{2}+t^{2}\left\|h_{2}\right\|^{2}}=\left\|x_{2}\right\| \sqrt{1+2 t \frac{x_{2}^{T} h_{2}}{\left\|x_{2}\right\|^{2}}+t^{2} \frac{\left\|h_{2}\right\|^{2}}{\left\|x_{2}\right\|^{2}}} .
$$

Therefore, using the fact that

$$
\sqrt{1+\epsilon}=1+\frac{1}{2} \epsilon-\frac{1}{8} \epsilon^{2}+o\left(\epsilon^{2}\right)
$$

we may obtain

$$
\left\|x_{2}+t h_{2}\right\|=\left\|x_{2}\right\|\left(1+t \frac{\alpha}{\left\|x_{2}\right\|}+\frac{1}{2} t^{2} \frac{\beta}{\left\|x_{2}\right\|^{2}}\right)+o\left(t^{2}\right),
$$

where

$$
\alpha=\frac{x_{2}^{T} h_{2}}{\left\|x_{2}\right\|}, \quad \beta=\left\|h_{2}\right\|^{2}-\frac{\left(x_{2}^{T} h_{2}\right)^{2}}{\left\|x_{2}\right\|^{2}}=\left\|h_{2}\right\|^{2}-\alpha^{2}=h_{2}^{T} M_{x_{2}} h_{2},
$$

with

$$
M_{x_{2}}=I-\frac{x_{2} x_{2}^{T}}{\left\|x_{2}\right\|^{2}}
$$

Furthermore, from (13) and the fact that $(1+\epsilon)^{-1}=1-\epsilon+\epsilon^{2}+o\left(\epsilon^{2}\right)$, it follows that

$$
\left\|x_{2}+t h_{2}\right\|^{-1}=\left\|x_{2}\right\|^{-1}\left(1-t \frac{\alpha}{\left\|x_{2}\right\|}+\frac{1}{2} t^{2}\left(2 \frac{\alpha^{2}}{\left\|x_{2}\right\|^{2}}-\frac{\beta}{\left\|x_{2}\right\|^{2}}\right)+o\left(t^{2}\right)\right) .
$$

Combining Eqs. (13) and (14) then yields that

$$
\begin{aligned}
\frac{x_{2}+t h_{2}}{\left\|x_{2}+t h_{2}\right\|}= & \frac{x_{2}}{\left\|x_{2}\right\|}+t\left(\frac{h_{2}}{\left\|x_{2}\right\|}-\frac{\alpha}{\left\|x_{2}\right\|} \frac{x_{2}}{\left\|x_{2}\right\|}\right) \\
& +\frac{1}{2} t^{2}\left(\left(2 \frac{\alpha^{2}}{\left\|x_{2}\right\|^{2}}-\frac{\beta}{\left\|x_{2}\right\|^{2}}\right) \frac{x_{2}}{\left\|x_{2}\right\|}-2 \frac{h_{2}}{\left\|x_{2}\right\|} \frac{\alpha}{\left\|x_{2}\right\|}\right)+o\left(t^{2}\right) \\
= & \frac{x_{2}}{\left\|x_{2}\right\|}+t M_{x_{2}} \frac{h_{2}}{\left\|x_{2}\right\|} \\
& +\frac{1}{2} t^{2}\left(3 \frac{h_{2}^{T} x_{2} x_{2}^{T} h_{2}}{\left\|x_{2}\right\|^{4}} \frac{x_{2}}{\left\|x_{2}\right\|}-\frac{\left\|h_{2}\right\|^{2}}{\left\|x_{2}\right\|^{2}} \frac{x_{2}}{\left\|x_{2}\right\|}-2 \frac{h_{2} h_{2}^{T}}{\left\|x_{2}\right\|^{2}} \frac{x_{2}}{\left\|x_{2}\right\|}\right)+o\left(t^{2}\right) .
\end{aligned}
$$

In addition, from (13), we have the following equalities

$$
\begin{aligned}
& f\left(x_{1}+t h_{1}-\left\|x_{2}+t h_{2}\right\|\right) \\
= & f\left(x_{1}+t h_{1}-\left(\left\|x_{2}\right\|\left(1+t \frac{\alpha}{\left\|x_{2}\right\|}+\frac{1}{2} t^{2} \frac{\beta}{\left\|x_{2}\right\|^{2}}\right)+o\left(t^{2}\right)\right)\right) \\
= & f\left(\lambda_{1}+t\left(h_{1}-\alpha\right)-\frac{1}{2} t^{2} \frac{\beta}{\left\|x_{2}\right\|}+o\left(t^{2}\right)\right) \\
= & f\left(\lambda_{1}\right)+t f^{\prime}\left(\lambda_{1}\right)\left(h_{1}-\alpha\right)+\frac{1}{2} t^{2}\left(-f^{\prime}\left(\lambda_{1}\right) \frac{\beta}{\left\|x_{2}\right\|}+f^{\prime \prime}\left(\lambda_{1}\right)\left(h_{1}-\alpha\right)^{2}\right)+o\left(t^{2}\right)
\end{aligned}
$$


and

$$
\begin{aligned}
& f\left(x_{1}+t h_{1}+\left\|x_{2}+t h_{2}\right\|\right) \\
= & f\left(\lambda_{2}+t\left(h_{1}+\alpha\right)+\frac{1}{2} t^{2} \frac{\beta}{\left\|x_{2}\right\|}+o\left(t^{2}\right)\right) \\
= & f\left(\lambda_{2}\right)+t f^{\prime}\left(\lambda_{2}\right)\left(h_{1}+\alpha\right)+\frac{1}{2} t^{2}\left(f^{\prime}\left(\lambda_{2}\right) \frac{\beta}{\left\|x_{2}\right\|}+f^{\prime \prime}\left(\lambda_{2}\right)\left(h_{1}+\alpha\right)^{2}\right)+o\left(t^{2}\right) .
\end{aligned}
$$

For $i=0,1,2$, we define

$$
a^{(i)}=\frac{f^{(i)}\left(\lambda_{2}\right)-f^{(i)}\left(\lambda_{1}\right)}{\lambda_{2}-\lambda_{1}}, \quad b^{(i)}=\frac{f^{(i)}\left(\lambda_{2}\right)+f^{(i)}\left(\lambda_{1}\right)}{2}, \quad c^{(i)}=\frac{f^{(i)}\left(\lambda_{2}\right)-f^{(i)}\left(\lambda_{1}\right)}{2},
$$

where $f^{(i)}$ means the $i$-th derivative of $f$ and $f^{(0)}$ is the same as the original $f$. Then, by the Eqs. (16)-(18), it can be verified that

$$
\begin{aligned}
\Xi_{1} & =\frac{1}{2}\left(f\left(x_{1}+t h_{1}+\left\|x_{2}+t h_{2}\right\|\right)+f\left(x_{1}+t h_{1}-\left\|x_{2}+t h_{2}\right\|\right)\right) \\
& =b^{(0)}+t\left(b^{(1)} h_{1}+c^{(1)} \alpha\right)+\frac{1}{2} t^{2}\left(a^{(1)} \beta+b^{(2)}\left(h_{1}^{2}+\alpha^{2}\right)+2 c^{(2)} h_{1} \alpha\right)+o\left(t^{2}\right) \\
& =b^{(0)}+t\left(b^{(1)} h_{1}+c^{(1)} h_{2}^{T} \frac{x_{2}}{\left\|x_{2}\right\|}\right)+\frac{1}{2} t^{2} h^{T} A_{1}(x) h+o\left(t^{2}\right),
\end{aligned}
$$

where

$$
A_{1}(x)=\left[\begin{array}{cc}
b^{(2)} & c^{(2)} \frac{x_{2}^{T}}{\left\|x_{2}\right\|} \\
c^{(2)} \frac{x_{2}}{\left\|x_{2}\right\|} a^{(1)} I+\left(b^{(2)}-a^{(1)}\right) \frac{x_{2} x_{2}^{T}}{\left\|x_{2}\right\|^{2}}
\end{array}\right] .
$$

Note that in the above expression for $\Xi_{1}, b^{(0)}$ is exactly the first component of $f^{\text {soc }}(x)$ and $\left(b^{(1)} h_{1}+c^{(1)} h_{2}^{T} \frac{x_{2}}{\left\|x_{2}\right\|}\right)$ is the first component of $\nabla f^{\text {soc }}(x) h$. Using the same techniques again,

$$
\begin{aligned}
& \frac{1}{2}\left(f\left(x_{1}+t h_{1}+\left\|x_{2}+t h_{2}\right\|\right)-f\left(x_{1}+t h_{1}-\left\|x_{2}+t h_{2}\right\|\right)\right) \\
= & c^{(0)}+t\left(c^{(1)} h_{1}+b^{(1)} \alpha\right)+\frac{1}{2} t^{2}\left(b^{(1)} \frac{\beta}{\left\|x_{2}\right\|}+c^{(2)}\left(h_{1}^{2}+\alpha^{2}\right)+2 b^{(2)} h_{1} \alpha\right)+o\left(t^{2}\right) \\
= & c^{(0)}+t\left(c^{(1)} h_{1}+b^{(1)} \alpha\right)+\frac{1}{2} t^{2} h^{T} B(x) h+o\left(t^{2}\right),
\end{aligned}
$$

where

$$
B(x)=\left[\begin{array}{cc}
c^{(2)} & b^{(2)} \frac{x_{2}^{T}}{\left\|x_{2}\right\|} \\
b^{(2)} \frac{x_{2}}{\left\|x_{2}\right\|} c^{(2)} I+\left(\frac{b^{(1)}}{\left\|x_{2}\right\|}-c^{(2)}\right) M_{x_{2}}
\end{array}\right] .
$$

Using Eqs. (15) and (20), we obtain that

$$
\begin{aligned}
\Xi_{2} & =\frac{1}{2}\left(f\left(x_{1}+t h_{1}+\left\|x_{2}+t h_{2}\right\|\right)-f\left(x_{1}+t h_{1}-\left\|x_{2}+t h_{2}\right\|\right)\right) \frac{x_{2}+t h_{2}}{\left\|x_{2}+t h_{2}\right\|} \\
& =c^{(0)} \frac{x_{2}}{\left\|x_{2}\right\|}+t\left(\frac{x_{2}}{\left\|x_{2}\right\|}\left(c^{(1)} h_{1}+b^{(1)} \alpha\right)+c^{(0)} M_{x_{2}} \frac{h_{2}}{\left\|x_{2}\right\|}\right)+\frac{1}{2} t^{2} W+o\left(t^{2}\right),
\end{aligned}
$$


where

$$
\begin{aligned}
W= & \frac{x_{2}}{\left\|x_{2}\right\|} h^{T} B(x) h+2 M_{x_{2}} \frac{h_{2}}{\left\|x_{2}\right\|}\left(c^{(1)} h_{1}+b^{(1)} \alpha\right) \\
& +c^{(0)}\left(3 \frac{h_{2}^{T} x_{2} x_{2}^{T} h_{2}}{\left\|x_{2}\right\|^{4}} \frac{x_{2}}{\left\|x_{2}\right\|}-\frac{\left\|h_{2}\right\|^{2}}{\left\|x_{2}\right\|^{2}} \frac{x_{2}}{\left\|x_{2}\right\|}-2 \frac{h_{2} h_{2}^{T}}{\left\|x_{2}\right\|^{2}} \frac{x_{2}}{\left\|x_{2}\right\|}\right) .
\end{aligned}
$$

Now we denote

$$
\begin{aligned}
d & :=\frac{b^{(1)}-a^{(0)}}{\left\|x_{2}\right\|}=\frac{2\left(b^{(1)}-a^{(0)}\right)}{\lambda_{2}-\lambda_{1}}, \quad U:=h^{T} C(x) h \\
V & :=2 \frac{c^{(1)} h_{1}+b^{(1)} \alpha}{\left\|x_{2}\right\|}-c^{(0)} 2 \frac{x_{2}^{T} h_{2}}{\left\|x_{2}\right\|^{3}}=2 a^{(1)} h_{1}+2 d \frac{x_{2}^{T} h_{2}}{\left\|x_{2}\right\|},
\end{aligned}
$$

where

$$
C(x):=\left[\begin{array}{cc}
c^{(2)} & \left(b^{(2)}-a^{(1)}\right) \frac{x_{2}^{T}}{\left\|x_{2}\right\|} \\
\left(b^{(2)}-a^{(1)}\right) \frac{x_{2}}{\left\|x_{2}\right\|} \mathrm{d} I+\left(c^{(2)}-3 d\right) \frac{x_{2} x_{2}^{T}}{\left\|x_{2}\right\|^{2}}
\end{array}\right] .
$$

Then $U$ can be further recast as

$$
U=h^{T} B(x) h+c^{(0)} 3 \frac{h_{2}^{T} x_{2} x_{2}^{T} h_{2}}{\left\|x_{2}\right\|^{4}}-c^{(0)} \frac{\left\|h_{2}\right\|^{2}}{\left\|x_{2}\right\|^{2}}-2 \frac{x_{2}^{T} h_{2}}{\left\|x_{2}\right\|^{2}}\left(c^{(1)} h_{1}+b^{(1)} \alpha\right) .
$$

Consequently,

$$
W=\frac{x_{2}}{\left\|x_{2}\right\|} U+h_{2} V .
$$

We next consider the case where $x_{2}=0$ and $x_{2}+t h_{2} \neq 0$. By definition (5),

$$
\begin{aligned}
f^{\mathrm{soc}}(x+t h) & =\frac{f\left(x_{1}+t\left(h_{1}-\left\|h_{2}\right\|\right)\right)}{2}\left[\begin{array}{c}
1 \\
-\frac{h_{2}}{\left\|h_{2}\right\|}
\end{array}\right]+\frac{f\left(x_{1}+t\left(h_{1}+\left\|h_{2}\right\|\right)\right)}{2}\left[\begin{array}{c}
1 \\
\frac{h_{2}}{\left\|h_{2}\right\|}
\end{array}\right] \\
& =\left[\frac{\frac{f\left(x_{1}+t\left(h_{1}-\left\|h_{2}\right\|\right)\right)+f\left(x_{1}+t\left(h_{1}+\left\|h_{2}\right\|\right)\right)}{2}}{2}\right] .
\end{aligned}
$$

Using the Taylor expansion of $f$ at $x_{1}$, we can obtain that

$$
\begin{aligned}
& \frac{1}{2}\left[f\left(x_{1}+t\left(h_{1}-\left\|h_{2}\right\|\right)\right)+f\left(x_{1}+t\left(h_{1}+\left\|h_{2}\right\|\right)\right)\right] \\
= & f\left(x_{1}\right)+t f^{(1)}\left(x_{1}\right) h_{1}+\frac{1}{2} t^{2} f^{(2)}\left(x_{1}\right) h^{T} h+o\left(t^{2}\right), \\
& \frac{1}{2}\left[f\left(x_{1}+t\left(h_{1}-\left\|h_{2}\right\|\right)\right)-f\left(x_{1}+t\left(h_{1}+\left\|h_{2}\right\|\right)\right)\right] \\
= & t f^{(1)}\left(x_{1}\right) h_{2}+\frac{1}{2} t^{2} f^{(2)}\left(x_{1}\right) 2 h_{1} h_{2}+o\left(t^{2}\right) .
\end{aligned}
$$

Therefore,

$$
f^{\mathrm{soc}}(x+t h)=f^{\mathrm{soc}}(x)+t f^{(1)}\left(x_{1}\right) h+\frac{1}{2} t^{2} f^{(2)}\left(x_{1}\right)\left[\begin{array}{c}
h^{T} h \\
2 h_{1} h_{2}
\end{array}\right] .
$$


Thus, under this case, we have that

$$
A_{1}(x)=f^{(2)}\left(x_{1}\right) I, \quad A_{i}(x)=f^{(2)}\left(x_{1}\right)\left(\begin{array}{cc}
0 & \bar{e}_{i-1}^{T} \\
\bar{e}_{i-1} & \mathbf{0}
\end{array}\right) \quad i=2, \ldots, n,
$$

where $\bar{e}_{j} \in \mathbb{R}^{n-1}$ is the vector whose $j$ th component is 1 and the others are 0 .

Summing up the above discussions, we may obtain the following conclusion.

Proposition 2.1 Let $f \in C^{(2)}(J)$ with $J$ being an open interval in $\mathbb{R}$ and $\operatorname{dom} f^{\mathrm{soc}} \subseteq \mathbb{R}^{n}$. Then, for given $x \in \operatorname{dom} f^{\mathrm{soc}}, h \in \mathbb{R}^{n}$ and any sufficiently small $t>0$,

$$
f^{\mathrm{soc}}(x+t h)=f^{\mathrm{soc}}(x)+t \nabla f^{\mathrm{soc}}(x) h+\frac{1}{2} t^{2}\left[\begin{array}{c}
h^{T} A_{1}(x) h \\
h^{T} A_{2}(x) h \\
\vdots \\
h^{T} A_{n}(x) h
\end{array}\right]+o\left(t^{2}\right),
$$

where $\nabla f^{\mathrm{soc}}(x)$ and $A_{i}(x), i=1,2, \ldots, n$ are given by (10) and (25) if $x_{2}=0$; and otherwise $\nabla f^{\text {soc }}(x)$ and $A_{1}(x)$ are given by (9) and (19), respectively, and for $i \geq 2$,

$$
A_{i}(x)=C(x) \frac{x_{2 i}}{\left\|x_{2}\right\|}+B_{i}(x)
$$

where

$$
B_{i}(x)=v e_{i}^{T}+e_{i} v^{T}, \quad v=\left[a^{(1)} d \frac{x_{2}^{T}}{\left\|x_{2}\right\|}\right]^{T} .
$$

From Proposition 4.3 of [5] and Proposition 2.1, we readily have the following result.

Proposition 2.2 Let $f \in C^{(2)}(J)$ with $J$ being an open interval in $\mathbb{R}$ and $\operatorname{dom} f^{\mathrm{soc}} \subseteq \mathbb{R}^{n}$. Then, $f$ is SOC-convex if and only if for any $x \in \operatorname{dom} f^{\mathrm{soc}}$ and $h \in \mathbb{R}^{n}$, the vector

$$
\left[\begin{array}{c}
h^{T} A_{1}(x) h \\
h^{T} A_{2}(x) h \\
\vdots \\
h^{T} A_{n}(x) h
\end{array}\right] \in \mathcal{K}^{n}
$$

\section{Characterizations of SOC-monotone functions}

Now we are ready to show our main result concerning the characterization of SOC-monotone functions. We need the following technical lemmas for the proof. The first one is so-called S-Lemma whose proof can be found in $[16,18]$.

Lemma 3.1 Let $A, B$ be symmetric matrices and $y^{T} A y>0$ for some $y$. Then, the implication $\left[z^{T} A z \geq 0 \Rightarrow z^{T} B z \geq 0\right]$ is valid if and only if $B \succeq \lambda A$ for some $\lambda \geq 0$.

Lemma 3.2 Given $\theta \in \mathbb{R}, a \in \mathbb{R}^{n-1}$, and a symmetric matrix $A \in \mathbb{R}^{n \times n}$. Let $\mathcal{B}^{n-1}:=$ $\left\{z \in \mathbb{R}^{n-1} \mid\|z\| \leq 1\right\}$. Then, the following results hold:

(a) For any $h \in \mathcal{K}^{n}$, Ah $\in \mathcal{K}^{n}$ is equivalent to $A\left[\begin{array}{l}1 \\ z\end{array}\right] \in \mathcal{K}^{n}$ for any $z \in \mathcal{B}^{n-1}$.

(b) For any $z \in \mathcal{B}^{n-1}, \theta+a^{T} z \geq 0$ is equivalent to $\theta \geq\|a\|$. 
(c) If $A=\left[\begin{array}{ll}\theta & a^{T} \\ a & H\end{array}\right]$ with $H$ being an $(n-1) \times(n-1)$ symmetric matrix, then for any $h \in \mathcal{K}^{n}, A h \in \mathcal{K}^{n}$ is equivalent to $\theta \geq\|a\|$ and there exists $\lambda \geq 0$ such that the matrix

$$
\left[\begin{array}{cc}
\theta^{2}-\|a\|^{2}-\lambda & \theta a^{T}-a^{T} H \\
\theta a-H^{T} a & a a^{T}-H^{T} H+\lambda I
\end{array}\right] \succeq O .
$$

Proof (a) For any $h \in \mathcal{K}^{n}$, suppose that $A h \in \mathcal{K}^{n}$. Let $h=\left[\begin{array}{l}1 \\ z\end{array}\right]$ where $z \in \mathcal{B}^{n-1}$. Then $h \in \mathcal{K}^{n}$ and the desired result follows. For the other direction, if $h=0$, the conclusion is obvious. Now let $h:=\left(h_{1}, h_{2}\right)$ be any nonzero vector in $\mathcal{K}^{n}$. Then, $h_{1}>0$ and $\left\|h_{2}\right\| \leq h_{1}$. Consequently, $\frac{h_{2}}{h_{1}} \in \mathcal{B}^{n-1}$ and $A\left[\begin{array}{l}1 \\ \frac{h_{2}}{h_{1}}\end{array}\right] \in \mathcal{K}^{n}$. Since $\mathcal{K}^{n}$ is a cone, we have

$$
h_{1} A\left[\begin{array}{l}
1 \\
\frac{h_{2}}{h_{1}}
\end{array}\right]=A h \in \mathcal{K}^{n}
$$

(b) For $z \in \mathcal{B}^{n-1}$, suppose $\theta+a^{T} z \geq 0$. If $a=0$, then the result is clear since $\theta \geq 0$. If $a \neq 0$, let $z:=-a /\|a\|$. Clearly, $z \in \mathcal{B}^{n-1}$ and hence $\theta+\frac{-a^{T} a}{\|a\|} \geq 0$ which gives $\theta-\|a\| \geq 0$. For the other direction, the result follows from the Cauchy Schwarz inequality:

$$
\theta+a^{T} z \geq \theta-\|a\| \cdot\|z\| \geq \theta-\|a\| \geq 0 .
$$

(c) From part (a), $A h \in \mathcal{K}^{n}$ for any $h \in \mathcal{K}^{n}$ is equivalent to $A\left[\begin{array}{l}1 \\ z\end{array}\right] \in \mathcal{K}^{n}$ for any $z \in \mathcal{B}^{n-1}$. Notice that

$$
A\left[\begin{array}{l}
1 \\
z
\end{array}\right]=\left[\begin{array}{ll}
\theta & a^{T} \\
a & H
\end{array}\right]\left[\begin{array}{l}
1 \\
z
\end{array}\right]=\left[\begin{array}{l}
\theta+a^{T} z \\
a+H z
\end{array}\right] .
$$

Then, $A h \in \mathcal{K}^{n}$ for any $h \in \mathcal{K}^{n}$ is equivalent to the following two things:

$$
\theta+a^{T} z \geq 0, \text { for any } z \in \mathcal{B}^{n-1}
$$

and

$$
(a+H z)^{T}(a+H z) \leq\left(\theta+a^{T} z\right)^{2}, \text { for any } z \in \mathcal{B}^{n-1} .
$$

By part (b), (26) is equivalent to $\theta \geq\|a\|$. Now, we write the expression of (27) as below:

$$
z^{T}\left(a a^{T}-H^{T} H\right) z+2\left(\theta a^{T}-a^{T} H\right) z+\theta^{2}-a^{T} a \geq 0, \text { for any } z \in \mathcal{B}^{n-1},
$$

which can be further simplified as

$$
\left[\begin{array}{ll}
1 & z^{T}
\end{array}\right]\left[\begin{array}{cc}
\theta^{2}-\|a\|^{2} & \theta a^{T}-a^{T} H \\
\theta a-H^{T} a & a a^{T}-H^{T} H
\end{array}\right]\left[\begin{array}{l}
1 \\
z
\end{array}\right] \geq 0, \quad \text { for any } z \in \mathcal{B}^{n-1} .
$$

Observe that $z \in \mathcal{B}^{n-1}$ is the same as

$$
\left[\begin{array}{ll}
1 & z^{T}
\end{array}\right]\left[\begin{array}{cc}
1 & 0 \\
0 & -I
\end{array}\right]\left[\begin{array}{l}
1 \\
z
\end{array}\right] \geq 0
$$


Thus, by applying the S-Lemma (Lemma 3.1), there exists $\lambda \geq 0$ such that

$$
\left[\begin{array}{cc}
\theta^{2}-\|a\|^{2} & \theta a^{T}-a^{T} H \\
\theta a-H^{T} a & a a^{T}-H^{T} H
\end{array}\right]-\lambda\left[\begin{array}{cc}
1 & 0 \\
0 & -I
\end{array}\right] \succeq O
$$

This completes the proof of part (c).

Theorem 3.1 Let $f \in C^{(1)}(J)$ with $J$ being an open interval and $\operatorname{dom} f^{\mathrm{soc}} \subseteq \mathbb{R}^{n}$. Then,

(i) when $n=2, f$ is SOC-monotone if and only if $f^{\prime}(\tau) \geq 0$ for any $\tau \in J$;

(ii) when $n \geq 3, f$ is SOC-monotone if and only if the $2 \times 2$ matrix

$$
\left[\begin{array}{cc}
f^{(1)}\left(t_{1}\right) & \frac{f\left(t_{2}\right)-f\left(t_{1}\right)}{t_{2}-t_{1}} \\
\frac{f\left(t_{2}\right)-f\left(t_{1}\right)}{t_{2}-t_{1}} & f^{(1)}\left(t_{2}\right)
\end{array}\right] \succeq O \text { for all } t_{1}, t_{2} \in J .
$$

Proof By the definition of SOC-monotonicity, $f$ is SOC-monotone if and only if

$$
f^{\mathrm{soc}}(x+h)-f^{\mathrm{soc}}(x) \in \mathcal{K}^{n}
$$

for any $x \in \operatorname{dom} f^{\text {soc }}$ and $h \in \mathcal{K}^{n}$ such that $x+h \in \operatorname{dom} f^{\text {soc }}$. By the first-order Taylor expansion of $f^{\text {soc }}$, i.e.,

$$
f^{\mathrm{soc}}(x+h)=f^{\mathrm{soc}}(x)+\nabla f^{\mathrm{soc}}(x+t h) h \quad \text { for some } t \in(0,1),
$$

it is clear that (28) is equivalent to $\nabla f^{\mathrm{soc}}(x+t h) h \in \mathcal{K}^{n}$ for any $x \in \operatorname{dom} f^{\mathrm{soc}}$ and $h \in \mathcal{K}^{n}$ such that $x+h \in \operatorname{dom} f^{\text {soc }}$, and some $t \in(0,1)$. Let $y:=x+t h=\mu_{1} v^{(1)}+\mu_{2} v^{(2)}$ for such $x, h$ and $t$. We next proceed the arguments by the two cases of $y_{2} \neq 0$ and $y_{2}=0$.

Case (1): $y_{2} \neq 0$. Under this case, we notice that

$$
\nabla f^{\mathrm{soc}}(y)=\left[\begin{array}{cc}
\theta & a^{T} \\
a & H
\end{array}\right]
$$

where

$$
\theta=\tilde{b}^{(1)}, \quad a=\tilde{c}^{(1)} \frac{y_{2}}{\left\|y_{2}\right\|}, \quad \text { and } H=\tilde{a}^{(0)} I+\left(\tilde{b}^{(1)}-\tilde{a}^{(0)}\right) \frac{y_{2} y_{2}^{T}}{\left\|y_{2}\right\|^{2}},
$$

with

$$
\tilde{a}^{(0)}=\frac{f\left(\mu_{2}\right)-f\left(\mu_{1}\right)}{\mu_{2}-\mu_{1}}, \quad \tilde{b}^{(1)}=\frac{f^{\prime}\left(\mu_{2}\right)+f^{\prime}\left(\mu_{1}\right)}{2}, \quad \tilde{c}^{(1)}=\frac{f^{\prime}\left(\mu_{2}\right)-f^{\prime}\left(\mu_{1}\right)}{2} .
$$

In addition, we also observe that

$$
\theta^{2}-\|a\|^{2}=\left(\tilde{b}^{(1)}\right)^{2}-\left(\tilde{c}^{(1)}\right)^{2}, \quad \theta a^{T}-a^{T} H=0
$$

and

$$
a a^{T}-H^{T} H=-\left(\tilde{a}^{(0)}\right)^{2} I+\left(\left(\tilde{c}^{(1)}\right)^{2}-\left(\tilde{b}^{(1)}\right)^{2}+\left(\tilde{a}^{(0)}\right)^{2}\right) \frac{y_{2} y_{2}^{T}}{\left\|y_{2}\right\|^{2}} .
$$

Thus, by Lemma 3.2, $f$ is SOC-monotone if and only if

(a) $\tilde{b}^{(1)} \geq\left|\tilde{c}^{(1)}\right|$; 
(b) and there exists $\lambda \geq 0$ such that the matrix

$$
\left[\begin{array}{cc}
\left(\tilde{b}^{(1)}\right)^{2}-\left(\tilde{c}^{(1)}\right)^{2}-\lambda & 0 \\
0 & \left(\lambda-\left(\tilde{a}^{(0)}\right)^{2}\right) I+\left(\left(\tilde{c}^{(1)}\right)^{2}-\left(\tilde{b}^{(1)}\right)^{2}+\left(\tilde{a}^{(0)}\right)^{2}\right) \frac{y_{2} y_{2}^{T}}{\left\|y_{2}\right\|^{2}}
\end{array}\right] \succeq O .
$$

When $n=2$, (a) together with (b) is equivalent to saying that $f^{\prime}\left(\mu_{1}\right) \geq 0$ and $f^{\prime}\left(\mu_{2}\right) \geq 0$. Then we conclude that $f$ is SOC-monotone if and only if $f^{\prime}(\tau) \geq 0$ for any $\tau \in J$.

When $n \geq 3$, (b) is equivalent to saying that $\left(\tilde{b}^{(1)}\right)^{2}-\left(\tilde{c}^{(1)}\right)^{2}=\lambda \geq 0$ and $\lambda-\left(\tilde{a}^{(0)}\right)^{2} \geq 0$, i.e., $\left(\tilde{b}^{(1)}\right)^{2}-\left(\tilde{c}^{(1)}\right)^{2} \geq\left(\tilde{a}^{(0)}\right)^{2}$. Therefore, (a) together with (b) is equivalent to

$$
\left[\begin{array}{cc}
f^{(1)}\left(\mu_{1}\right) & \frac{f\left(\mu_{2}\right)-f\left(\mu_{1}\right)}{\mu_{2}-\mu_{1}} \\
\frac{f\left(\mu_{2}\right)-f\left(\mu_{1}\right)}{\mu_{2}-\mu_{1}} & f^{(1)}\left(\mu_{2}\right)
\end{array}\right] \succeq O
$$

for any $x \in \mathbb{R}^{n}, h \in \mathcal{K}^{n}$ such that $x+h \in \operatorname{dom} f^{\mathrm{soc}}$, and some $t \in(0,1)$. Thus, we conclude that $f$ is SOC-monotone if and only if

$$
\left[\begin{array}{cc}
f^{(1)}\left(t_{1}\right) & \frac{f\left(t_{2}\right)-f\left(t_{1}\right)}{t_{2}-t_{1}} \\
\frac{f\left(t_{2}\right)-f\left(t_{1}\right)}{t_{2}-t_{1}} & f^{(1)}\left(t_{2}\right)
\end{array}\right] \succeq O \text { for all } t_{1}, t_{2} \in J .
$$

Case (2): $y_{2}=0$. Now we have $\mu_{1}=\mu_{2}$ and $\nabla f^{\mathrm{soc}}(y)=f^{(1)}\left(\mu_{1}\right) I=f^{(1)}\left(\mu_{2}\right) I$. Hence, $f$ is SOC-monotone is equivalent to $f^{(1)}\left(\mu_{1}\right) \geq 0$, which is also equivalent to

$$
\left[\begin{array}{cc}
f^{(1)}\left(\mu_{1}\right) & \frac{f\left(\mu_{2}\right)-f\left(\mu_{1}\right)}{\mu_{2}-\mu_{1}} \\
\frac{f\left(\mu_{2}\right)-f\left(\mu_{1}\right)}{\mu_{2}-\mu_{1}} & f^{(1)}\left(\mu_{2}\right)
\end{array}\right] \succeq O
$$

since $f^{(1)}\left(\mu_{1}\right)=f^{(1)}\left(\mu_{2}\right)$ and $\frac{f\left(\mu_{2}\right)-f\left(\mu_{1}\right)}{\mu_{2}-\mu_{1}}=f^{(1)}\left(\mu_{1}\right)=f^{(1)}\left(\mu_{2}\right)$ by the Taylor formula and $\mu_{1}=\mu_{2}$. Thus, similar to Case (1), the conclusion also holds under this case.

From Theorem 3.1 and [11, Theorem 6.6.36], we immediately have the following results.

Corollary 3.1 Let $f \in C^{(1)}(J)$ with $J$ being an open interval in $\mathbb{R}$. Then,

(a) $f$ is SOC-monotone of order $n \geq 3$ if and only if it is 2-matrix monotone, and $f$ is SOC-monotone of order $n \leq 2$ if it is 2-matrix monotone.

(b) Suppose that $n \geq 3$ and $f$ is SOC-monotone of order $n$. Then, $f^{\prime}\left(t_{0}\right)=0$ for some $t_{0} \in J$ if and only if $f(\cdot)$ is a constant function on $J$.

Note that the SOC-monotonicity of order 2 does not imply the 2-matrix monotonicity. For example, $f(t)=t^{2}$ is SOC-monotone of order 2 on $(0,+\infty)$ by Example 3.2 (a) in [5], but by [11, Theorem 6.6.36] we can verify that it is not 2-matrix monotone. Corollary 3.1 (a) implies that a continuously differentiable function defined on an open interval must be SOC-monotone if it is 2-matrix monotone. In addition, from the following proposition, we also have that the compound of two simple SOC-monotone functions is SOC-monotone.

Proposition 3.1 If $f: J_{1} \rightarrow J$ and $g: J \rightarrow \mathbb{R}$ with $J_{1}, J \subseteq \mathbb{R}$ are SOC-monotone on $J_{1}$ and $J$, respectively, then the function $g \circ f: J_{1} \rightarrow \mathbb{R}$ is SOC-monotone on $J$.

Proof It is easy to verify that for all $x, y \in \mathbb{R}^{n}, x \succeq \mathcal{K}^{n} y$ if and only if $\lambda_{i}(x) \geq \lambda_{i}(y)$ with $i=1$, 2. In addition, $g$ is monotone on $J$ since it is SOC-monotone. From the two facts, we immediately obtain the result. 


\section{Characterizations of SOC-convex functions}

In this section, we exploit Peirce decomposition to derive some characterizations for SOCconvex functions. Let $f \in C^{(2)}(J)$ with $J$ being an open interval in $\mathbb{R}$ and $\operatorname{dom} f^{\text {soc }} \subseteq \mathbb{R}^{n}$.

For any $x \in \operatorname{dom} f^{\mathrm{soc}}$ and $h \in \mathbb{R}^{n}$, if $x_{2}=0$, then from Proposition 2.1 we have that

$$
\left[\begin{array}{c}
h^{T} A_{1}(x) h \\
h^{T} A_{2}(x) h \\
\vdots \\
h^{T} A_{n}(x) h
\end{array}\right]=f^{(2)}\left(x_{1}\right)\left[\begin{array}{c}
h^{T} h \\
2 h_{1} h_{2}
\end{array}\right] .
$$

Since $\left(h^{T} h, 2 h_{1} h_{2}\right) \in \mathcal{K}^{n}$, from Proposition 2.2 it follows that $f$ is SOC-convex if and only if $f^{(2)}\left(x_{1}\right) \geq 0$. By the arbitrariness of $x_{1}, f$ is SOC-convex if and only if $f$ is convex on $J$.

In what follows, we assume that $x_{2} \neq 0$. Let $x=\lambda_{1}(x) u_{x}^{(1)}+\lambda_{2}(x) u_{x}^{(2)}$, where $u_{x}^{(1)}$ and $u_{x}^{(2)}$ are given by (4) with $\bar{x}_{2}=\frac{x_{2}}{\left\|x_{2}\right\|}$. Let $u_{x}^{(i)}=\left(0, v_{2}^{(i)}\right)$ for $i=3, \ldots, n$, where $v_{2}^{(3)}, \ldots, v_{2}^{(n)}$ is any orthonormal set of vectors that span the subspace of $\mathbb{R}^{n-2}$ orthogonal to $x_{2}$. It is easy to verify that the vectors $u_{x}^{(1)}, u_{x}^{(2)}, u_{x}^{(3)}, \ldots, u_{x}^{(n)}$ are linearly independent. Hence, for any given $h=\left(h_{1}, h_{2}\right) \in \mathbb{R} \times \mathbb{R}^{n-1}$, there exists $\mu_{i}, i=1,2, \ldots, n$ such that

$$
h=\mu_{1} \sqrt{2} u_{x}^{(1)}+\mu_{2} \sqrt{2} u_{x}^{(2)}+\sum_{i=3}^{n} \mu_{i} u_{x}^{(i)} .
$$

From (19), we can verify that $b^{(2)}+c^{(2)}$ and $b^{(2)}-c^{(2)}$ are the eigenvalues of $A_{1}(x)$ with $u_{x}^{(2)}$ and $u_{x}^{(1)}$ being the corresponding eigenvectors, and $a^{(1)}$ is the eigenvalue of multiplicity $n-2$ with $u_{x}^{(i)}=\left(0, v_{2}^{(i)}\right)$ for $i=3, \ldots, n$ being the corresponding eigenvectors. Therefore,

$$
\begin{aligned}
h^{T} A_{1}(x) h & =\mu_{1}^{2}\left(b^{(2)}-c^{(2)}\right)+\mu_{2}^{2}\left(b^{(2)}+c^{(2)}\right)+a^{(1)} \sum_{i=3}^{n} \mu_{i}^{2} \\
& =f^{(2)}\left(\lambda_{1}\right) \mu_{1}^{2}+f^{(2)}\left(\lambda_{2}\right) \mu_{2}^{2}+a^{(1)} \mu^{2},
\end{aligned}
$$

where

$$
\mu^{2}=\sum_{i=3}^{n} \mu_{i}^{2}
$$

Similarly, we can verify that $c^{(2)}+b^{(2)}-a^{(1)}$ and $c^{(2)}-b^{(2)}+a^{(1)}$ are the eigenvalues of

$$
\left[\begin{array}{cc}
c^{(2)} & \left(b^{(2)}-a^{(1)}\right) \frac{x_{2}^{T}}{\left\|x_{2}\right\|} \\
\left(b^{(2)}-a^{(1)}\right) \frac{x_{2}}{\left\|x_{2}\right\|} \mathrm{d} I+\left(c^{(2)}-d\right) \frac{x_{2} x_{2}^{T}}{\left\|x_{2}\right\|^{2}}
\end{array}\right]
$$

with $u_{x}^{(2)}$ and $u_{x}^{(1)}$ being the corresponding eigenvectors, and $d$ is the eigenvalue of multiplicity $n-2$ with $u_{x}^{(i)}=\left(0, v_{2}^{(i)}\right)$ for $i=3, \ldots, n$ being the corresponding eigenvectors. Notice that $C(x)$ in (22) can be decomposed the sum of the above matrix and

$$
\left[\begin{array}{cc}
0 & 0 \\
0 & -2 d \frac{x_{2} x_{2}^{T}}{\left\|x_{2}\right\|^{2}}
\end{array}\right] .
$$


Consequently,

$$
h^{T} C(x) h=\mu_{1}^{2}\left(c^{(2)}-b^{(2)}+a^{(1)}\right)+\mu_{2}^{2}\left(c^{(2)}+b^{(2)}-a^{(1)}\right)-d\left(\mu_{2}-\mu_{1}\right)^{2}+d \mu^{2} .
$$

In addition, by the definition of $B_{i}(x)$, it is easy to compute that

$$
h^{T} B_{i}(x) h=\sqrt{2} h_{2, i-1}\left(\mu_{1}\left(a^{(1)}-d\right)+\mu_{2}\left(a^{(1)}+d\right)\right),
$$

where $h_{2 i}=\left(h_{21}, \ldots, h_{2, n-1}\right)$. From Eqs. (30)-(32) and the definition of $A_{i}(x)$ in Proposition 2.1, we thus have

$$
\begin{aligned}
\sum_{i=2}^{n}\left(h^{T} A_{i}(x) h\right)^{2}= & {\left[h^{T} C(x) h\right]^{2}+2\left\|h_{2}\right\|^{2}\left(\mu_{1}\left(a^{(1)}-d\right)+\mu_{2}\left(a^{(1)}+d\right)\right)^{2} } \\
& +2\left(\mu_{2}-\mu_{1}\right) h^{T} C(x) h\left(\mu_{1}\left(a^{(1)}-d\right)+\mu_{2}\left(a^{(1)}+d\right)\right) \\
= & {\left[h^{T} C(x) h\right]^{2}+2\left(\frac{1}{2}\left(\mu_{2}-\mu_{1}\right)^{2}+\mu^{2}\right)\left(\mu_{1}\left(a^{(1)}-d\right)+\mu_{2}\left(a^{(1)}+d\right)\right)^{2} } \\
& +2\left(\mu_{2}-\mu_{1}\right) h^{T} C(x) h\left(\mu_{1}\left(a^{(1)}-d\right)+\mu_{2}\left(a^{(1)}+d\right)\right) \\
= & {\left[h^{T} C(x) h+\left(\mu_{2}-\mu_{1}\right)\left(\mu_{1}\left(a^{(1)}-d\right)+\mu_{2}\left(a^{(1)}+d\right)\right)\right]^{2} } \\
& +2 \mu^{2}\left(\mu_{1}\left(a^{(1)}-d\right)+\mu_{2}\left(a^{(1)}+d\right)\right)^{2} \\
= & {\left[-f^{(2)}\left(\lambda_{1}\right) \mu_{1}^{2}+f^{(2)}\left(\lambda_{2}\right) \mu_{2}^{2}+d \mu^{2}\right]^{2} } \\
& +2 \mu^{2}\left(\mu_{1}\left(a^{(1)}-d\right)+\mu_{2}\left(a^{(1)}+d\right)\right)^{2} .
\end{aligned}
$$

On the other hand, by Proposition 2.2, $f$ is SOC-convex if and only if

$$
A_{1}(x) \succeq O \text { and } \sum_{i=2}^{n}\left(h^{T} A_{i}(x) h\right)^{2} \leq\left(h^{T} A_{1}(x) h\right)^{2} .
$$

From (30) and (33)-(45), we have that $f$ is SOC convex if and only if $A_{1}(x) \succeq O$ and

$$
\begin{aligned}
& {\left[-f^{(2)}\left(\lambda_{1}\right) \mu_{1}^{2}+f^{(2)}\left(\lambda_{2}\right) \mu_{2}^{2}+d \mu^{2}\right]^{2}+2 \mu^{2}\left(\mu_{1}\left(a^{(1)}-d\right)+\mu_{2}\left(a^{(1)}+d\right)\right)^{2}} \\
& \leq\left[f^{(2)}\left(\lambda_{1}\right) \mu_{1}^{2}+f^{(2)}\left(\lambda_{2}\right) \mu_{2}^{2}+a^{(1)} \mu^{2}\right]^{2} .
\end{aligned}
$$

When $n=2$, it is clear that $\mu=0$. Then, $f$ is SOC-convex if and only if

$$
A_{1}(x) \succeq O \text { and } f^{(2)}\left(\lambda_{1}\right) f^{(2)}\left(\lambda_{2}\right) \geq 0 .
$$

From the previous discussions, we know that $b^{(2)}-c^{(2)}=f^{(2)}\left(\lambda_{1}\right), b^{(2)}+c^{(2)}=f^{(2)}\left(\lambda_{2}\right)$ and $a^{(1)}=\frac{f^{(1)}\left(\lambda_{2}\right)-f^{(1)}\left(\lambda_{1}\right)}{\lambda_{2}-\lambda_{1}}$ are all eigenvalues of $A_{1}(x)$. Thus, $f$ is SOC-convex if and only if

$$
f^{(2)}\left(\lambda_{2}\right) \geq 0, \quad f^{(2)}\left(\lambda_{1}\right) \geq 0, \quad f^{(1)}\left(\lambda_{2}\right) \geq f^{(1)}\left(\lambda_{1}\right),
$$

which by the arbitrariness of $x$ is equivalent to saying that $f$ is convex on $J$.

When $n \geq 3$, if $\mu=0$, then from the discussions above, we know that $f$ is SOC-convex if and only if $f$ is convex. If $\mu \neq 0$, without loss of generality, we assume that $\mu^{2}=1$. Then the inequality (46) above is equivalent to

$$
\begin{aligned}
& 4 f^{(2)}\left(\lambda_{1}\right) f^{(2)}\left(\lambda_{2}\right) \mu_{1}^{2} \mu_{2}^{2}+\left(a^{(1)}\right)^{2}-d^{2} \\
& +2 f^{(2)}\left(\lambda_{2}\right) \mu_{2}^{2}\left(a^{(1)}-d\right)+2 f^{(2)}\left(\lambda_{1}\right) \mu_{1}^{2}\left(a^{(1)}+d\right) \\
& -2\left(\mu_{1}^{2}\left(a^{(1)}-d\right)^{2}+\mu_{2}^{2}\left(a^{(1)}+d\right)^{2}+2 \mu_{1} \mu_{2}\left(\left(a^{(1)}\right)^{2}-d^{2}\right)\right) \\
& \geq 0 \quad \text { for any } \mu_{1}, \mu_{2} .
\end{aligned}
$$


Now we show that $A_{1}(x) \succeq O$ and (36) holds if and only if $f$ is convex on $J$ and

$$
\begin{aligned}
& f^{(2)}\left(\lambda_{1}\right)\left(a^{(1)}+d\right) \geq\left(a^{(1)}-d\right)^{2}, \\
& f^{(2)}\left(\lambda_{2}\right)\left(a^{(1)}-d\right) \geq\left(a^{(1)}+d\right)^{2} .
\end{aligned}
$$

Indeed, if $f$ is convex on $J$, then by the discussions above $A_{1}(x) \succeq O$ clearly holds. If the inequalities (37) and (38) hold, then by the convexity of $f$ we have $a^{(1)} \geq|d|$. If $\mu_{1} \mu_{2} \leq 0$, then we readily have the inequality (36). If $\mu_{1} \mu_{2}>0$, then using $a^{(1)} \geq|d|$ yields that

$$
f^{(2)}\left(\lambda_{1}\right) f^{(2)}\left(\lambda_{2}\right) \mu_{1}^{2} \mu_{2}^{2} \geq\left(a^{(1)}\right)^{2}-d^{2} .
$$

Combining with Eqs. (37)-(38) thus leads to the inequality (36). On the other hand, if $A_{1}(x) \succeq$ $O$, then $f$ must be convex on $J$ by the discussions above, whereas if the inequality (36) holds for any $\mu_{1}, \mu_{2}$, then by letting $\mu_{1}=\mu_{2}=0$ yields that

$$
a^{(1)} \geq|d| \text {. }
$$

Using the inequality (39) and letting $\mu_{1}=0$ in (36) then yields (37), whereas using (39) and letting $\mu_{2}=0$ in (36) leads to (38). Thus, when $n \geq 3, f$ is SOC-convex if and only if $f$ is convex on $J$ and (37) and (38) hold. We notice that (37) and (38) are equivalent to

$$
\frac{1}{2} f^{(2)}\left(\lambda_{1}\right) \frac{\left[f\left(\lambda_{1}\right)-f\left(\lambda_{2}\right)+f^{(1)}\left(\lambda_{2}\right)\left(\lambda_{2}-\lambda_{1}\right)\right]}{\left(\lambda_{2}-\lambda_{1}\right)^{2}} \geq \frac{\left[f\left(\lambda_{2}\right)-f\left(\lambda_{1}\right)-f^{(1)}\left(\lambda_{1}\right)\left(\lambda_{2}-\lambda_{1}\right)\right]^{2}}{\left(\lambda_{2}-\lambda_{1}\right)^{4}}
$$

and

$$
\frac{1}{2} f^{(2)}\left(\lambda_{2}\right) \frac{\left[f\left(\lambda_{2}\right)-f\left(\lambda_{1}\right)-f^{(1)}\left(\lambda_{1}\right)\left(\lambda_{2}-\lambda_{1}\right)\right]}{\left(\lambda_{2}-\lambda_{1}\right)^{2}} \geq \frac{\left[f\left(\lambda_{1}\right)-f\left(\lambda_{2}\right)+f^{(1)}\left(\lambda_{2}\right)\left(\lambda_{2}-\lambda_{1}\right)\right]^{2}}{\left(\lambda_{2}-\lambda_{1}\right)^{4}} .
$$

Therefore, $f$ is SOC-convex if and only if $f$ is convex on $J$, and

$$
\begin{aligned}
& \frac{1}{2} f^{(2)}\left(t_{0}\right) \frac{\left[f\left(t_{0}\right)-f(t)-f^{(1)}(t)\left(t_{0}-t\right)\right]}{\left(t_{0}-t\right)^{2}} \geq \frac{\left[f(t)-f\left(t_{0}\right)-f^{(1)}\left(t_{0}\right)\left(t-t_{0}\right)\right]^{2}}{\left(t_{0}-t\right)^{4}} \\
& \forall t_{0}, t \in J .
\end{aligned}
$$

Summing up the above analysis, we can characterize the SOC-convexity as follows.

Theorem 4.1 Let $f \in C^{(2)}(J)$ with $J$ being an open interval in $\mathbb{R}$ and $\operatorname{dom} f^{\mathrm{soc}} \subseteq \mathbb{R}^{n}$. If $n=2$, then $f$ is SOC-convex if and only if $f$ is convex; if $n \geq 3$, then $f$ is SOC-convex if and only if $f$ is convex and the inequality (40) holds for any $t_{0}, t \in J$.

By the formulas of divided differences, it is not hard to verify that $f$ is convex on $J$ and (40) holds for any $t_{0}, t \in J$ if and only if

$$
\left[\begin{array}{cc}
\triangle^{2} f\left(t_{0}, t_{0}, t_{0}\right) & \triangle^{2} f\left(t_{0}, t, t_{0}\right) \\
\triangle^{2} f\left(t, t_{0}, t_{0}\right) & \triangle^{2} f\left(t, t, t_{0}\right)
\end{array}\right] \succeq O .
$$

This, together with Theorem 4.1 and [11, Theorem 6.6.52], leads to the following results.

Corollary 4.1 Let $f \in C^{(2)}(J)$ with $J$ being an open interval in $\mathbb{R}$ and $\operatorname{dom} f^{\mathrm{soc}} \subseteq \mathbb{R}^{n}$. Then, $f$ is SOC-convex of order $n \geq 3$ if and only if it is 2-matrix convex, and $f$ is SOC-convex of order $n \leq 2$ if it is 2-matrix convex.

Corollary 4.1 implies that, if $f$ is a twice continuously differentiable function defined on an open interval $J$ and 2-matrix convex, then it must be SOC-convex. Similar to Corollary 3.1 (a), when $f$ is SOC-convex of order 2, it may not be 2-matrix convex. For example, 
$f(t)=t^{3}$ is SOC-convex of order 2 on $(0,+\infty)$ by Example 3.3 (c) of [5], but it is easy to verify that (41) does not hold for this function, and consequently, $f$ is not 2-matrix convex. Using Corollary 4.1, we may prove that Conjecture 4.2 in [5] does not hold in general.

Corollary 4.2 Let $f:[0,+\infty) \rightarrow[0,+\infty)$ be continuous. If $f$ is SOC-concave, then $f$ is $S O C$-monotone. Conversely, if $f$ is SOC-monotone, then it may not be SOC-concave.

Proof The first part has been shown in [5]. We next consider the second part. Consider

$$
f(t)=-\cot \left(-\frac{\pi}{2}(1+t)^{-1}+\pi\right), \quad t \in[0,+\infty) .
$$

Notice that $-\cot (t)$ is SOC-monotone on $[\pi / 2, \pi)$, whereas $-\frac{\pi}{2}(1+t)^{-1}$ is SOC-monotone on $[0,+\infty)$. Hence, their compound function $f(t)$ is SOC-monotone on $[0,+\infty)$. However, $-f(t)$ does not satisfy the inequality (40) for all $t \in(0,+\infty)$. For example, when $t_{1}=7.7$ and $t_{2}=7.6$, the left hand side of (40) equals 0.0080 , whereas the right hand side equals 27.8884. This shows that $f(t)=-\cot (t)$ is not SOC-concave of order $n \geq 3$.

Particularly, by Corollary 4.1 and [9, Theorem 2.3], we can establish the following characterizations for SOC-convex functions.

Corollary 4.3 Let $f \in C^{(4)}(J)$ with $J$ being an open interval in $\mathbb{R}$ and $\operatorname{dom} f^{\mathrm{soc}} \subseteq \mathbb{R}^{n}$. If $f^{(2)}(t)>0$ for every $t \in J$, then $f$ is SOC-convex of order $n$ with $n \geq 3$ if and only if one of the following conditions holds.

(a) For every $t \in J$, the $2 \times 2$ matrix

$$
\left[\begin{array}{ll}
\frac{f^{(2)}(t)}{2} & \frac{f^{(3)}(t)}{6} \\
\frac{f^{(3)}(t)}{6} & \frac{f^{(4)}(t)}{24}
\end{array}\right] \succeq O .
$$

(b) There is a positive concave function c on I such that $f^{(2)}(t)=c(t)^{-3}$ for every $t \in J$.

(c) There holds that

$$
\begin{aligned}
& \frac{\left[f\left(t_{0}\right)-f(t)-f^{(1)}(t)\left(t_{0}-t\right)\right]}{\left(t_{0}-t\right)^{2}} \frac{\left[f(t)-f\left(t_{0}\right)-f^{(1)}\left(t_{0}\right)\left(t-t_{0}\right)\right]}{\left(t_{0}-t\right)^{2}} \\
& \leq \frac{1}{4} f^{(2)}\left(t_{0}\right) f^{(2)}(t) .
\end{aligned}
$$

Moreover, $f$ is also SOC-convex of order 2 under one of the above conditions.

Proof For completeness, we here present their proofs. Notice that $f$ is convex on $J$. By Corollary 4.1, it suffices to prove the following equivalence:

$$
\text { (40) } \Longleftrightarrow \text { assertion (a) } \Longleftrightarrow \text { assertion (b) } \Longleftrightarrow \text { assertion (c). }
$$

$(40) \Rightarrow$ assertion (a): From the previous discussions, we know that (40) is equivalent to (37) and (38). We expand (37) using Taylor's expansion at $\lambda_{1}$ to the fourth order and get

$$
\frac{3}{4} f^{(2)}\left(\lambda_{1}\right) f^{(4)}\left(\lambda_{1}\right) \geq\left(f^{(3)}\left(\lambda_{1}\right)\right)^{2} .
$$

We do the same for the inequality (38) at $\lambda_{2}$ and get the inequality

$$
\frac{3}{4} f^{(2)}\left(\lambda_{2}\right) f^{(4)}\left(\lambda_{2}\right) \geq\left(f^{(3)}\left(\lambda_{2}\right)\right)^{2} .
$$


The above two inequalities are precisely

$$
\frac{3}{4} f^{(2)}(t) f^{(4)}(t) \geq\left(f^{(3)}(t)\right)^{2} \quad \forall t \in J
$$

which is clearly equivalent to saying that the $2 \times 2$ matrix in (a) is positive semidefinite.

Assertion (a) $\Rightarrow$ assertion (b): Take $c(t)=\left[f^{(2)}(t)\right]^{-1 / 3}$ for $t \in J$. Then $c$ is a positive function and $f^{(2)}(t)=c(t)^{-3}$. By twice differentiation, we obtain

$$
f^{(4)}(t)=12 c(t)^{-5}\left[c^{\prime}(t)(t)\right]^{2}-3 c(t)^{-4} c^{\prime \prime}(t) .
$$

Substituting the last equality into the matrix in (a) then yields that

$$
-\frac{1}{16} c(t)^{-7} c^{\prime \prime}(t) \geq 0
$$

which, together with $c(t)>0$ for every $t \in J$, implies that $c$ is concave.

Assertion (b) $\Rightarrow$ assertion (c): We first prove the following fact: if $f^{(2)}(t)$ is strictly positive for every $t \in J$ and the function $c(t)=\left[f^{(2)}(t)\right]^{-1 / 3}$ is concave on $J$, then

$$
\frac{\left[f\left(t_{0}\right)-f(t)-f^{(1)}(t)\left(t_{0}-t\right)\right]}{\left(t_{0}-t\right)^{2}} \leq \frac{1}{2} f^{(2)}\left(t_{0}\right)^{1 / 3} f^{(2)}(t)^{2 / 3} \quad \forall t_{0}, t \in J .
$$

Indeed, using the concavity of the function $c$, it follows that

$$
\begin{aligned}
\frac{\left[f\left(t_{0}\right)-f(t)-f^{(1)}(t)\left(t_{0}-t\right)\right]}{\left(t_{0}-t\right)^{2}} & =\int_{0}^{1} \int_{0}^{u_{1}} f^{(2)}\left[t+u_{2}\left(t_{0}-t\right)\right] \mathrm{d} u_{2} \mathrm{~d} u_{1} \\
& \left.=\int_{0}^{1} \int_{0}^{u_{1}} c\left(\left(1-u_{2}\right) t+u_{2} t_{0}\right)\right)^{-3} \mathrm{~d} u_{2} \mathrm{~d} u_{1} \\
& \leq \int_{0}^{1} \int_{0}^{u_{1}}\left(\left(1-u_{2}\right) c(t)+u_{2} c\left(t_{0}\right)\right)^{-3} \mathrm{~d} u_{2} \mathrm{~d} u_{1} .
\end{aligned}
$$

Notice that $g(t)=1 / t(t>0)$ has the second-order derivative $g^{(2)}(t)=2 / t^{3}$. Hence,

$$
\begin{aligned}
\frac{\left[f\left(t_{0}\right)-f(t)-f^{(1)}(t)\left(t_{0}-t\right)\right]}{\left(t_{0}-t\right)^{2}} & \leq \frac{1}{2} \int_{0}^{1} \int_{0}^{u_{1}} g^{(2)}\left(\left(1-u_{2}\right) c(t)+u_{2} c\left(t_{0}\right)\right) \mathrm{d} u_{2} \mathrm{~d} u_{1} \\
& =\frac{1}{2}\left(\frac{g\left(c\left(t_{0}\right)\right)-g(c(t))}{\left(c\left(t_{0}\right)-c(t)\right)^{2}}-\frac{g^{(1)}(c(t))}{c\left(t_{0}\right)-c(t)}\right) \\
& =\frac{1}{2 c\left(t_{0}\right) c(t) c(t)} \\
& =\frac{1}{2} f^{(2)}\left(t_{0}\right)^{1 / 3} f^{(2)}(t)^{2 / 3},
\end{aligned}
$$

which implies the inequality (45). Now exchanging $t_{0}$ with $t$ in (45), we obtain

$$
\frac{\left[f(t)-f\left(t_{0}\right)-f^{(1)}\left(t_{0}\right)\left(t-t_{0}\right)\right]}{\left(t_{0}-t\right)^{2}} \leq \frac{1}{2} f^{(2)}(t)^{1 / 3} f^{(2)}\left(t_{0}\right)^{2 / 3} \quad \forall t, t_{0} \in J .
$$

Since $f$ is convex on $J$ by the given assumption, the left hand sides of the inequalities (45) and (46) are nonnegative, and their product satisfies the inequality of (43).

Assertion (c) $\Rightarrow$ (40): We introducing a function $F: J \rightarrow \mathbb{R}$ defined by

$$
F(t)=\frac{1}{2} f^{(2)}\left(t_{0}\right)\left[f\left(t_{0}\right)-f(t)-f^{(1)}(t)\left(t_{0}-t\right)\right]-\frac{\left[f(t)-f\left(t_{0}\right)-f^{(1)}\left(t_{0}\right)\left(t-t_{0}\right)\right]^{2}}{\left(t_{0}-t\right)^{2}}
$$


if $t \neq t_{0}$, and otherwise $F\left(t_{0}\right)=0$. We next prove that $F$ is nonnegative on $J$. It is easy to verify that such $F(t)$ is differentiable on $J$, and moreover,

$$
\begin{aligned}
F^{\prime}(t)= & \frac{1}{2} f^{(2)}\left(t_{0}\right) f^{(2)}(t)\left(t-t_{0}\right) \\
& -2\left(t-t_{0}\right)^{-2}\left[f(t)-f\left(t_{0}\right)-f^{(1)}\left(t_{0}\right)\left(t-t_{0}\right)\right]\left(f^{(1)}(t)-f^{(1)}\left(t_{0}\right)\right) \\
& +2\left(t-t_{0}\right)^{-3}\left[f(t)-f\left(t_{0}\right)-f^{(1)}\left(t_{0}\right)\left(t-t_{0}\right)\right]^{2} \\
= & \frac{1}{2} f^{(2)}\left(t_{0}\right) f^{(2)}(t)\left(t-t_{0}\right) \\
& -2\left(t-t_{0}\right)^{-3}\left[f(t)-f\left(t_{0}\right)-f^{(1)}\left(t_{0}\right)\left(t-t_{0}\right)\right]\left[f\left(t_{0}\right)-f(t)-f^{(1)}(t)\left(t_{0}-t\right)\right] \\
= & 2\left(t-t_{0}\right)\left[\frac{1}{4} f^{(2)}\left(t_{0}\right) f^{(2)}(t)-\left(t-t_{0}\right)^{-4}\left(f(t)-f\left(t_{0}\right)-f^{(1)}\left(t_{0}\right)\left(t-t_{0}\right)\right)\right. \\
& \left.\quad\left(f\left(t_{0}\right)-f(t)-f^{(1)}(t)\left(t_{0}-t\right)\right)\right] .
\end{aligned}
$$

Using the inequality in part (c), we can verify that $F(t)$ has a minimum value 0 at $t=t_{0}$, and therefore, $F(t)$ is nonnegative on $J$. This implies the inequality (40).

\section{Examples and applications}

The SOC-monotonicity and the SOC-convexity are often involved in the solution methods of convex SOCPs; for example, the proximal-like methods proposed in [15]. In this section, we focus on their applications in the augmented Lagrangian methods for the convex SOCP:

$$
\begin{array}{cl}
\min & f(\zeta) \\
\text { s.t. } & A \zeta+b \succeq_{\mathcal{K}^{n}} 0,
\end{array}
$$

where $f: \mathbb{R}^{n} \rightarrow \mathbb{R}$ is a convex function, $A \in \mathbb{R}^{n \times m}$ has the full column rank, and $b \in \mathbb{R}^{n}$.

Let $\varphi:(b,+\infty) \rightarrow \mathbb{R}$ be a function with the following favorable properties:

(i) $\varphi$ is strictly convex, strictly monotone increasing and twice continuously differentiable on its domain $\operatorname{dom} \varphi:=(b,+\infty)$ with $-\infty<b<0$;

(ii) $\varphi(0)=0, \varphi^{\prime}(0)=1$ and $\lim _{t \rightarrow b} \varphi^{\prime}(t)=\infty, \lim _{t \rightarrow+\infty} \varphi^{\prime}(t)=0$;

(iii) $\lim _{\varepsilon \rightarrow 0} \varepsilon \varphi(t / \varepsilon)=0$ for any $t>0$;

(iv) $\varphi$ is SOC-monotone on $(b,+\infty)$ and $\varphi$ is SOC-concave on $(b,+\infty)$.

For any $\varepsilon>0$, we use such $\varphi$ to define the function $\varphi_{\varepsilon}:(b \varepsilon,+\infty) \rightarrow \mathbb{R}$ by

$$
\varphi_{\varepsilon}(t)=\varepsilon \varphi(t / \varepsilon) .
$$

Then, by the properties (i)-(iii) and the SOC-monotonicity of $\varphi$, we can rewrite (47) as

$$
\begin{array}{ll}
\min & f(\zeta) \\
\text { s.t. } \varphi_{\varepsilon}^{\text {soc }}(A \zeta+b) \succeq_{\mathcal{K}^{n}} 0 .
\end{array}
$$

Moreover, by the SOC-convexity of $\varphi$, it is not difficult to verify that (49) is also a convex programming problem. Consequently, its Lagrangian, given by

$$
L_{\varepsilon}(x, y):=f(x)-\left\langle y, \varphi_{\varepsilon}{ }^{\mathrm{soc}}(A x+b)\right\rangle
$$

is convex for any $x \in \operatorname{dom} \varphi_{\varepsilon}{ }^{\text {soc }}$. Similar to the augmented Lagrangian method for nonlinear convex programming in nonnegative orthant cone, we can design the augmented Lagrangian 
method for (47) by solving a sequence of convex subproblems

$$
\min _{x \in \operatorname{dom} \varphi_{\varepsilon}} L_{\varepsilon}\left(x, y^{k}\right) \quad \text { with } y^{k} \succeq_{\mathcal{K}^{n}} 0 .
$$

In what follows, we employ the characterizations established in the last two sections to present some examples of SOC-monotone functions and SOC-convex functions, from which we can choose a suitable one to design the augmented Lagrangian method for (47).

Proposition 5.1 (a) For any fixed $\sigma \in \mathbb{R}$, the function $f(t)=\frac{1}{\sigma-t}$ is SOC-monotone on $(\sigma,+\infty)$.

(b) For any fixed $\sigma \in \mathbb{R}$, the function $f(t)=\sqrt{t-\sigma}$ is SOC-monotone on $[\sigma,+\infty)$.

(c) For any fixed $\sigma \in \mathbb{R}$, the function $f(t)=\ln (t-\sigma)$ is SOC-monotone on $(\sigma,+\infty)$.

(d) For any fixed $\sigma \geq 0$, the function $f(t)=\frac{t}{t+\sigma}$ is SOC-monotone on $(-\sigma,+\infty)$.

Proof (a) For any $t_{1}, t_{2} \in(\sigma,+\infty)$, it is clear that

$$
\left[\begin{array}{cc}
\frac{1}{\left(\sigma-t_{1}\right)^{2}} & \frac{1}{\left(\sigma-t_{2}\right)\left(\sigma-t_{1}\right)} \\
\frac{1}{\left(\sigma-t_{2}\right)\left(\sigma-t_{1}\right)} & \frac{1}{\left(\sigma-t_{2}\right)^{2}}
\end{array}\right] \succeq O
$$

From Theorem 3.1, we then obtain the desired result.

(b) If $x \succeq_{\mathcal{K}^{n}} \sigma e$, then $(x-\sigma e)^{1 / 2} \succeq_{\mathcal{K}^{n}} 0$. Thus, by Theorem 3.1, it suffices to show

$$
\left[\begin{array}{cc}
\frac{1}{2 \sqrt{t_{1}-\sigma}} & \frac{\sqrt{t_{2}-\sigma}-\sqrt{t_{1}-\sigma}}{t_{2}-t_{1}} \\
\frac{\sqrt{t_{2}-\sigma}-\sqrt{t_{1}-\sigma}}{t_{2}-t_{1}} & \frac{1}{2 \sqrt{t_{2}-\sigma}}
\end{array}\right] \succeq O \text { for any } t_{1}, t_{2}>0,
$$

which is equivalent to proving that

$$
\frac{1}{4 \sqrt{t_{1}-\sigma} \sqrt{t_{2}-\sigma}}-\frac{1}{\left(\sqrt{t_{2}-\sigma}+\sqrt{t_{1}-\sigma}\right)^{2}} \geq 0 .
$$

This inequality holds by $4 \sqrt{t_{1}-\sigma} \sqrt{t_{2}-\sigma} \leq\left(\sqrt{t_{2}-\sigma}+\sqrt{t_{1}-\sigma}\right)^{2}$ for any $t_{1}, t_{2} \in$ $(\sigma,+\infty)$.

(c) By Theorem 3.1, it suffices to prove that for any $t_{1}, t_{2} \in(\sigma,+\infty)$,

$$
\left[\begin{array}{cc}
\frac{1}{\left(t_{1}-\sigma\right)} & \frac{1}{\left(t_{2}-t_{1}\right)} \ln \left(\frac{t_{2}-\sigma}{t_{1}-\sigma}\right) \\
\frac{1}{\left(t_{2}-t_{1}\right)} \ln \left(\frac{t_{2}-\sigma}{t_{1}-\sigma}\right) & \frac{1}{\left(t_{2}-\sigma\right)}
\end{array}\right] \succeq O,
$$

which is equivalent to showing that

$$
\frac{1}{\left(t_{1}-\sigma\right)\left(t_{2}-\sigma\right)}-\left[\frac{1}{\left(t_{2}-t_{1}\right)} \ln \left(\frac{t_{2}-\sigma}{t_{1}-\sigma}\right)\right]^{2} \geq 0 .
$$

Notice that $\ln t \leq t-1(t>0)$, and hence it is easy to verify that

$$
\left[\frac{1}{\left(t_{2}-t_{1}\right)} \ln \left(\frac{t_{2}-\sigma}{t_{1}-\sigma}\right)\right]^{2} \leq \frac{1}{\left(t_{1}-\sigma\right)\left(t_{2}-\sigma\right)} .
$$

Consequently, the desired result follows. 
(d) Since for any fixed $\sigma \geq 0$ and any $t_{1}, t_{2} \in(-\sigma,+\infty)$, there holds that

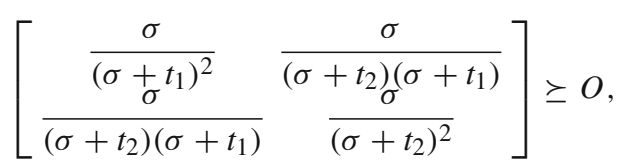

we immediately obtain the desired result from Theorem 3.1. The proof is complete.

Notice that Proposition 5.1 (a), (b) and (d) were established in [5] by the definition of SOC-monotonicity, and Proposition 5.1 (c) was shown in [15] by the definition of SOCmonotonicity and a transformation technique. However, we here use the characterization of Theorem 3.1 to convert their proofs into proving some simple inequalities on $\mathbb{R}$.

Proposition 5.2 (a) For any fixed $\sigma \in \mathbb{R}$, the function $f(t)=(t-\sigma)^{-r}$ with $r \geq 0$ is SOC-convex on $(\sigma,+\infty)$ if and only if $0 \leq r \leq 1$.

(b) For any fixed $\sigma \in \mathbb{R}$, the function $f(t)=(t-\sigma)^{r}$ with $r \geq 0$ is SOC-convex on $[\sigma,+\infty)$ if and only if $1 \leq r \leq 2$, and $f$ is SOC-concave on $[\sigma,+\infty)$ if and only if $0 \leq r \leq 1$.

(c) For any fixed $\sigma \in \mathbb{R}$, the function $f(t)=\ln (t-\sigma)$ is SOC-concave on $(\sigma,+\infty)$.

(d) For any fixed $\sigma \geq 0$, the function $f(t)=\frac{t}{t+\sigma}$ is SOC-concave on $(-\sigma,+\infty)$.

Proof (a) For any fixed $\sigma \in \mathbb{R}$, by a simple computation, we have that

$$
\left[\begin{array}{cc}
\frac{f^{(2)}(t)}{2} & \frac{f^{(3)}(t)}{6} \\
\frac{f^{(3)}(t)}{6} & \frac{f^{(4)}(t)}{24}
\end{array}\right]=\left[\begin{array}{cc}
\frac{r(r+1)(t-\sigma)^{-r-2}}{2} & \frac{r(r+1)(-r-2)(t-\sigma)^{-r-3}}{6} \\
\frac{r(r+1)(-r-2)(t-\sigma)^{-r-3}}{6} & \frac{r(r+1)(r+2)(r+3)(t-\sigma)^{-r-4}}{24}
\end{array}\right] .
$$

The sufficient and necessary condition for the above matrix being positive semidefinite is

$$
\frac{r^{2}(r+1)^{2}(r+2)(r+3)(t-\sigma)^{-2 r-6}}{24}-\frac{r^{2}(r+1)^{2}(r+2)^{2}(t-\sigma)^{-2 r-6}}{18} \geq 0,
$$

which is equivalent to requiring $0 \leq r \leq 1$. By Corollary 4.3, it then follows that $f$ is SOC-convex on $(\sigma,+\infty)$ if and only if $0 \leq r \leq 1$.

(b) For any fixed $\sigma \in \mathbb{R}$, by a simple computation, we have that

$$
\left[\begin{array}{cc}
\frac{f^{(2)}(t)}{2} & \frac{f^{(3)}(t)}{6} \\
\frac{f^{(3)}(t)}{6} & \frac{f^{(4)}(t)}{24}
\end{array}\right]=\left[\begin{array}{cc}
\frac{r(r-1)(t-\sigma)^{r-2}}{2} & \frac{r(r-1)(r-2)(t-\sigma)^{r-3}}{6} \\
\frac{r(r-1)(r-2)(t-\sigma)^{r-3}}{6} & \frac{r(r-1)(r-2)(r-3)(t-\sigma)^{r-4}}{24}
\end{array}\right] .
$$

The sufficient and necessary condition for the above matrix being positive semidefinite is

$$
\begin{array}{r}
r 1 \text { and } \frac{r^{2}(r-1)^{2}(r-2)(r-3)(t-\sigma)^{2 r-6}}{24} \\
-\frac{r^{2}(r-1)^{2}(r-2)^{2}(t-\sigma)^{2 r-6}}{18} \geq 0,
\end{array}
$$

whereas the sufficient and necessary condition for it being negative semidefinite is

$$
\begin{aligned}
0 \leq r \leq 1 \quad \text { and } & \frac{r^{2}(r-1)^{2}(r-2)(r-3)(t-\sigma) t^{2 r-6}}{24} \\
& -\frac{r^{2}(r-1)^{2}(r-2)^{2}(t-\sigma)^{2 r-6}}{18} \geq 0 .
\end{aligned}
$$


It is easily shown that (52) holds if and only if $1 \leq r \leq 2$, and (53) holds if and only if $0 \leq r \leq 1$. By Corollary 4.3, this shows that $f$ is SOC-convex on $(\sigma,+\infty)$ if and only if $1 \leq r \leq 2$, and $f$ is SOC-concave on $(\sigma,+\infty)$ if and only if $0 \leq r \leq 1$. This together with the definition of SOC-convexity then yields the desired result.

(c) Notice that for any $t>\sigma$, there always holds that

$$
-\left[\begin{array}{cc}
\frac{f^{(2)}(t)}{2} & \frac{f^{(3)}(t)}{6} \\
\frac{f^{(3)}(t)}{6} & \frac{f^{(4)}(t)}{24}
\end{array}\right]=\left[\begin{array}{cc}
\frac{1}{2(t-\sigma)^{2}} & -\frac{1}{3(t-\sigma)^{3}} \\
-\frac{1}{3(t-\sigma)^{3}} & \frac{1}{4(t-\sigma)^{4}}
\end{array}\right] \succeq O .
$$

Consequently, from Corollary 4.3 (a) we obtain that $f$ is SOC-concave on $(\sigma,+\infty)$.

(d) For any $t>-\sigma$, it is easy to compute that

$$
-\left[\begin{array}{cc}
\frac{f^{(2)}(t)}{2} & \frac{f^{(3)}(t)}{6} \\
\frac{f^{(3)}(t)}{6} & \frac{f^{(4)}(t)}{24}
\end{array}\right]=\left[\begin{array}{cc}
\frac{1}{(t+\sigma)^{3}} & -\frac{1}{(t+\sigma)^{4}} \\
-\frac{1}{(t+\sigma)^{4}} & \frac{1}{(t+\sigma)^{5}}
\end{array}\right] \succeq O .
$$

By Corollary 4.3, we then have that the function $f$ is SOC-concave on $(-\sigma,+\infty)$.

From Propositions 5.1 and 5.2, we see that the functions $\ln (1+t), 2 \sqrt{t+1}-2$ and $-\frac{1}{t+1}-1$ are SOC-monotone as well as SOC-concave. Furthermore, it is easy to verify that they also satisfy the properties (i)-(iii). Thus, with the three functions, we can design the augmented Lagrangian method for solving the convex SOCP (47), which in fact corresponds to the modified barrier functions method proposed by R. Ployak [17] for nonlinear programming over nonnegative orthant cones. We will leave the details of this method for a future research topic. In addition, we should point out that the SOC-monotonicity and the SOC-convexity often give a great help to establish some inequalities involved the partial order " $\succeq_{\mathcal{K}}$ ". For example, from Proposition 5.1 (b), we readily recover the Eq. 3.9 of [8, Proposition 3.4].

Acknowledgements The authors would like to thank two anonymous referees for their helpful comments and suggestions on the revision of this paper.

\section{References}

1. Aujla, J.S., Vasudeva, H.L.: Convex and monotone operator functions. Ann. Pol. Math. 62, 1-11 (1995)

2. Bhatia, R.: Matrix Analysis. Springer, New York (1997)

3. Bhatia, R., Parthasarathy, K.P.: Positive definite functions and operator inequalities. Bull. Lond. Math. Soc. 32, 214-228 (2000)

4. Brinkhuis, J., Luo, Z.-Q., Zhang, S.: Matrix convex functions with applications to weighted centers for semi-definite programming, submitted manuscript (2006)

5. Chen, J.-S.: The convex and monotone functions associated with second-order cone. Optimization 55, 363-385 (2006)

6. Chen, J.-S., Chen, X., Tseng, P.: Analysis of nonsmooth vector-valued functions associated with secondorder cones. Math. Program. 101, 95-117 (2004)

7. Faraut, J., Korányi, A.: Analysis on Symmetric Cones, Oxford Mathematical Monographs. Oxford University Press, New York (1994)

8. Fukushima, M., Luo, Z.-Q., Tseng, P.: Smoothing functions for second-order cone complementarity problems. SIAM J. Optim. 12, 436-460 (2002)

9. Hansen, F., Tomiyama, J.: Differential analysis of matrix convex functions. Linear Algebra Appl. 420, 102-116 (2007) 
10. Horn, R.A., Johnson, C.R.: Matrix Analysis. Cambridge University Press, Cambridge (1985)

11. Horn, R.A., Johnson, C.R.: Topics in Matrix Analysis. Cambridge University Press, Cambridge (1991)

12. Korányi, A.: Monotone functions on formally real Jordan algebras. Math. Ann. 269, 73-76 (1984)

13. Kwong, M.-K.: Some results on matrix monotone functions. Linear Algebra Appl. 118, 129-153 (1989)

14. Löwner, K.: Über monotone matrixfunktionen. Math. Z. 38, 177-216 (1934)

15. Pan, S.-H., Chen, J.-S.: A class of interior proximal-like algorithms for convex second-order cone programming, accepted by SIAM J. Optim. 19, 883-910 (2008)

16. Polik, I., Terlaky, T.: A Comprehensive Study of the S-Lemma. Advanced Optimization On-Line, Report No. 2004/14 (2004)

17. Polyak, R.: Modified barrier functions: theory and methods. Math. Program. 54, 177-222 (1992)

18. Roos, K.: http://www.isa.ewi.tudelft.nl/ roos/course/WI4218/SLemma.pdf

19. Sun, D., Sun, J.: Semismooth matrix valued functions. Math. Oper. Res. 27, 150-169 (2002)

20. Tseng, P.: Merit function for semidefinite complementarity problems. Math. Program. 83, 159185 (1998) 